\title{
IMAGING APPEARANCE OF NONGERMINOMA PEDIATRIC OVARIAN GERM CELL TUMORS DOES NOT DISCRIMINATE BENIGN FROM MALIGNANT HISTOLOGY
}

Deborah Billmire - Division of Pediatric Surgery, Indiana University, Indianapolis, Indiana Bryan Dicken - Division of Pediatric Surgery, University of Alberta, Edmonton, Alberta Canada Frederick Rescorla - Division of Pediatric Surgery, Indiana University, Indianapolis, Indiana Jonathan Ross - Department of Urology, Rush University, Chicago, Illinois Jin Piao - Childrens Oncology Group, Monrovia, California Li Huang - Childrens Oncology Group, Monrovia, California Mark Krailo Department of Research Preventive Medicine, University of Southern California, Monrovia, California

Farzana Pashankar - Department of Pediatrics, Yale School of Medicine, New Haven, Connecticut Lindsay Frazier - Department of Pediatrics, Harvard University, Dana Farber Cancer Institute, Boston, Massachusetts

On behalf of the Childrens Oncology Group

Address correspondence to:

Deborah F Billmire, MD Division of Pediatric Surgery. Riley Hospital for Children, suite 2500, 705 Riley Hospital Drive, Indianapolis, IN 46202. Phone 3172744682 Fax 317274 4461. email dbillmir@iupui.edu

Presented at the 2019 annual meeting of the American Pediatric Surgical Association in Boston, Massachusetts on May 21, 2019.

Abstract: 250 words

Manuscript: 1,934 words

Funding acknowledgment

This is the author's manuscript of the article published in final edited form as:

Billmire, D., Dicken, B., Rescorla, F., Ross, J., Piao, J., Huang, L., Krailo, M., Pashankar, F., \& Frazier, L. (2020). Imaging Appearance of Nongerminoma Pediatric Ovarian Germ Cell Tumors Does Not Discriminate Benign from Malignant Histology. Journal of Pediatric and Adolescent Gynecology. https://doi.org/10.1016/j.jpag.2020.11.014 
NCTN Operations Center Grant U10CA180886

NCTN Statistics \& Data Center Grant U10CA18089

St. Baldrick's Foundation 


\section{ABSTRACT}

Study objective: Pediatric ovarian neoplasms with imaging appearance suggestive of teratoma are often presumed to have low risk of malignancy. We assessed the pre-operative imaging appearance of pediatric malignant ovarian germ cell tumors (MOGCT) and the presence of associated teratoma in a series of MOGCT.

Design: Retrospective review of clinical and pathology data.

Setting: Multicenter trial for extra-cranial malignant germ cell tumors in children by the Children's Oncology Group (COG study AGCT0132) that Included yolk sac tumor, embryonal carcinoma and choriocarcinoma.

Participants: Girls age 0-18 years at enrollment with ovarian primary nonseminomatous malignant germ cell tumors.

Interventions: Review of data forms including prospectively collected surgical checklist documenting imaging characteristics of the tumor and review of pathology reports.

Main outcome measures: Description of imaging appearance and frequency of mixed histology with benign teratoma elements.

Results: 138 girls (11 months to 20 years) had primary ovarian tumors. Imaging appearance and pathology information were available for 133 patients. Among the 133 patients, tumor appearance was solid (10.5\%), solid with calcification (3.0\%), mixed cystic and solid (58.7\%), mixed cystic and solid with calcification (24.8\%) and unknown (3.0\%). 54\% had elements of teratoma in addition to malignant histology. 
Conclusions: Mixed cystic and solid appearance with or without calcification was seen in $83.5 \%$ of pediatric ovarian malignant germ cell tumors. Associated benign teratoma was common. The presence of a mixed cystic and solid appearance on pre-operative imaging should not dissuade the surgeon from obtaining preoperative serum markers and undertaking complete surgical staging.

Words 250

Keywords: ovarian germ cell tumors, ovary yolk sac tumor, teratoma

Ovarian masses are encountered at all ages in childhood and adolescence. In neonates, these masses are almost exclusively follicular cysts due to maternal hormone influence and most can be managed non-operatively [1]. Simple cysts beyond the neonatal period may present with pain due to hemorrhage or torsion but are clearly recognized by their simple wall, unilocular appearance and absence of any solid components. True neoplastic lesions account for approximately $73 \%$ of masses and $16-22 \%$ of these are malignant [2,3]. Since the majority of neoplastic lesions are benign teratomas (also called dermoid cysts) which have a mixed cystic and solid appearance and frequently have calcifications, surgeons may assume that this finding on imaging indicates a benign tumor. This leads to incomplete staging if a malignant tumor is present and may impact the subsequent need for chemotherapy. 
Recent attention has been focused on imaging characteristics that are predictive of malignancy, but non-neoplastic simple cysts are often included in these series and will have an impact on the risk predictor calculations. Diameter greater than $9 \mathrm{~cm}$ and solid appearance have been predictive of malignancy in recent pediatric series of ovarian masses but they have included simple cysts in the calculations. In addition, these reports are based on case series of ovarian masses from single institutions so the actual number of malignant cases is very small and the size cutoff is not absolute $[2,4$, 5]. Once simple cysts are excluded, the important dilemma is whether or not a neoplastic lesion may be malignant. The size range and imaging appearance of pediatric malignant ovarian germ cell tumors is unknown. This review was undertaken to summarize the preoperative ovarian imaging findings in the Children's Oncology Group study AGCT0132 of all the enrolled ovarian malignant germ cell tumors in children.

\section{PATIENTS AND METHODS}

The Children's Oncology Group study AGCT0132 for malignant extra cranial germ cell tumors enrolled patients between November 2003 and July 2011. Details have been reported elsewhere $[6,7,8]$. Data for this analysis is current to June 30,2018 . Required histology included yolk sac, embryonal carcinoma or choriocarcinoma. Pure germinoma and pure immature teratoma were not eligible. Low risk (stage I gonadal) patients were treated with surgery and surveillance with chemotherapy reserved for relapse. Intermediate risk (stage I-III extragonadal, stage II-IV testicular, stage II-III ovarian) patients were treated with chemotherapy in addition to surgery.

Primary ovarian malignant germ cell tumors were seen in 138 girls: 29 low risk (stage I), and 109 intermediate risk (stage II and III). A surgical checklist was required at enrollment for all patients. The ovarian surgical checklist included a request for the institution to provide an imaging description of the tumor as one of the following: purely cystic, cystic with calcifications, purely solid, solid with calcifications, mixture of solid and cystic components, mixture of solid and cystic components with calcifications, or unknown. Unknown appearance category was needed for those without preoperative imaging or with procedure done at an outside institution without submission of preoperative imaging. The surgical checklists and institutional and central review pathology reports were reviewed to evaluate imaging characteristics and histology for girls with ovarian germ cell tumors.

\section{RESULTS}

Completed surgical checklists, operative notes and pathology reports were available for 133 of 138 patients with characteristics as seen in Table 1 and form the basis for this review. Four intermediate risk patients and one low risk patient did not have a surgical checklist submitted and could not be included. As the study design excluded pure germinoma and there were no pure embryonal carcinomas, all patients had elevated tumor markers at diagnosis. Alpha-fetoprotein (AFP) was measured in all patients (median: $3394.5 \mathrm{ng} / \mathrm{ml}$, range: $2-4233000 \mathrm{ng} / \mathrm{ml}$ ) and beta human chorionogonadotrophin (BHCG) (median: $2 \mathrm{mIU} / \mathrm{mL}$, range: $0-999140 \mathrm{mIU} / \mathrm{mL}$ ). The imaging appearance according to the surgical checklists is described in Table 2 . No patients had purely cystic tumors with or without calcification. Overall, $58.7 \% \%$ of girls had an imaging appearance that was a mixture of solid and cystic components and $24.8 \%$ had a mixture of solid and cystic components with calcification. Seventy-two (54\%) had mixed histology that included teratoma (mature or immature) along with malignant histology (Table 3). Maximal tumor dimension ranged from 5.5 to $33.5 \mathrm{~cm}$ with a median of $16 \mathrm{~cm}$. 


\section{DISCUSSION}

This study was undertaken to evaluate the imaging characteristics of malignant nongerminoma ovarian germ cell tumors in pediatric and adolescent patients to determine if there were distinguishing features which could aid in preoperative discussion and planning of the operation. We found that over $80 \%$ had a mixed cystic and solid appearance which could easily be seen in a mature teratoma. We also noted that associated elements of benign teratoma were common and were seen in more than half of patients.

Ovarian masses in the pediatric age group are somewhat uncommon and have an overall risk of malignancy of 10-15\%. A recent paper [5] reviewed the imaging and marker characteristics of ovarian masses from two free standing pediatric institutions. They included all ovarian masses in patients less than 18 years of age undergoing surgical management of ovarian masses. That series included 185 patients with malignancy found in $11 \%$. Imaging findings in their series revealed simple cysts in 24 (12.9\%), solid masses in 34 (18.3\%) and heterogeneous masses in 127 (68.6\%). They did not distinguish whether calcification was present. All of the simple cysts were benign. Our series of exclusively malignant germ cell tumors also found no simple cysts on imaging. If the simple cystic lesions are excluded from the above series; the distribution of solid and heterogeneous appearance was 34/158 $(21.5 \%)$ solid tumors and $127 / 158(80.3 \%)$ heterogeneous masses. This distribution of imaging appearance is similar to our series of patients with malignant germ cell tumors in whom we noted $13.5 \%$ solid and $84.9 \%$ heterogeneous (mixed) masses. In addition, although risk factors for malignancy included size greater than $9 \mathrm{~cm}$ in greatest dimension, the malignant tumors in this series ranged in size from 5.5 to $33.5 \mathrm{~cm}$. These results indicate that imaging characteristics and size alone do not differentiate benign from malignant ovarian masses. It was disappointing to note on review of the operative notes in this series that there were several instances where the surgeon commented that the mass appeared to be classic for teratoma and/or failed to do full tumor staging. Mention of preoperative tumor markers was seldom included in the operative notes. It should also be noted that not all malignant germ cell tumors will have elevated serum markers. In all instances ovarian masses that are not simple cysts should be approached with caution and pre-operative investigation including tumor markers must be completed. Full pediatric surgical staging should always be undertaken for ovarian masses regardless of tumor marker findings.

Ovarian masses in the pediatric and adolescent age group are managed by a variety of surgical specialists. These factors make the experience for any individual surgeon limited both by experience and by training and contribute to the failure to undertake complete surgical staging at the primary procedure. The finding of cystic areas or calcifications on preoperative imaging may lead to the assumption that the mass is a benign teratoma and a limited procedure is done. Malignancy is not discovered until the pathology has been assessed and the opportunity for appropriate staging has been lost. The increasing and appropriate emphasis on ovarian sparing procedures to protect fertility in this age group has led to attempts to risk stratify for malignancy based on preoperative assessment. Recent reviews of single institution series of ovarian masses in children have evaluated preoperative tumor marker and imaging findings to aid in risk stratification of malignancy. As none of these classifications are $100 \%$ sensitive or specific, there is a possibility of under-staging by omitting required steps, or of upstaging by deliberately violating the tumor capsule of a malignant ovarian tumor during the surgical 
procedure. As an example, failure to collect peritoneal fluid or do a complete assessment of the peritoneal cavity may lead to missed pathology. In the previous Pediatric Oncology Group/Children's Cancer Study Group (POG/CCSG) intergroup study that formed the basis for the revised COG guidelines, 5 girls with otherwise stage I tumors were accurately recognized to be stage III based on peritoneal cytology alone confirming the importance of complete assessment [9]. Furthermore, surgical technique aimed at reducing mass size by planned puncture, or by an accidental capsule entry or rupture during manipulation for a presumed benign lesion; will upstage a patient who might otherwise be a candidate for surgery and surveillance. Laparoscopic enucleation of ovarian masses has been shown to result in a higher rupture rate of the lesion than open technique [10].

The current pediatric surgical ovarian germ cell tumor guidelines used by the Childrens Oncology Group (COG) were published in 2004 [9]. Despite their simplicity, compliance with the guidelines remains a significant problem. A 15-year retrospective study by Oltmann et al in 2010 [4] revealed only 24\% compliance with COG guidelines; and a similar 22-year retrospective study by Madenci et al in 2016 [11] showed $27 \%$ compliance with guidelines, without any change in performance after the 2004 guidelines were published. The pediatric guidelines are less extensive than the adult FIGO guidelines in terms of tissue sampling, but remain critical in accurate assessment and treatment planning. Inspection and documentation of all defined parameters in the operative note are absolute requirements for confirmation of stage I status and the possibility that a surveillance strategy, rather than chemotherapy, can be recommended. "Not documented" has to be considered as not having been done. In the surgery and surveillance arm of the AGCT0132 study for stage I ovarian tumors, relapse occurred in 9 of 21 girls who were confirmed as stage I by central review; but relapse was seen in 3 of 4 girls who were assigned as stage I by the enrolling institution but had incomplete staging on central review [6]. Overall survival for the stage I tumors was $96 \%$ at 4 years.

Limitations of this study include the inclusion of only malignant nongerminoma ovarian tumors. A strength of this study is the large number of malignant tumors with prospectively documented imaging appearance that could not be obtained in a single institution review.

It is recommended that all pediatric and adolescent ovarian neoplasms be approached with the concern that malignant histology may be present, regardless of tumor size or appearance, and that the COG guidelines for staging be completed. The guidelines include collection of peritoneal fluid for cytology, inspection and documentation of omentum, peritoneal surfaces, lymph nodes and opposite ovary. Biopsy of any abnormal findings is required. The tumor should be removed without violation of the tumor capsule in the peritoneal cavity. These tasks are easily accomplished in all patients with minimal expenditure of time and little to no morbidity. Preoperative CT imaging provides guidance to aid in identifying suspicious, enlarged nodes that should be biopsied. A low yield of positive peritoneal cytology should be expected [12], but the information is of high importance. A laparoscopic approach does not preclude the steps of the staging procedure, and inspection of the peritoneal surfaces may even be more easily accomplished. The ovarian mass should be excised with care to avoid rupture of the tumor capsule. Tumors with elevated markers should be treated with oophorectomy. Tumors with negative markers may be considered for an ovary sparing approach but complete performance and documentation of the staging procedure is imperative and should always be done. Care should be taken to minimize the risk of tumor spill when excising the mass from the ovarian parenchyma as this not only upstages malignant tumors but also increases the risk of recurrence for benign teratomas [10]. 
All authors have no conflicts of interest. 


\section{REFERENCES}

1. Brandt ML, Helmrath MA: Ovarian cysts in infants and children. Semin Pediatr Surg 2005; 14(2):78

2. Papic JC, Finnell SM, Slaven JE, et al: Predictors of ovarian malignancy in children: overcoming clinical barriers of ovarian preservation. J Pediatr Surg 2014; 49:144

3. Lawrence $A E$, Fallat $M E$, Hewitt $G$, et al: Understanding the value of tumor markers in pediatric ovarian neoplasms. J Pediatr Surg 2020; 55:122

4. Oltmann SC, Garcia NM, Barber R, et al: Pediatric ovarian malignancies: how efficacious are current staging practices? J Pediatr Surg 2010; 45:1096

5. Madenci AL, Levine BS, Laufer MR, et al: Preoperative risk stratification of children with ovarian tumors. J Pediatr Surg 2016; 51(9):1507

6. Billmire DF, Cullen JW, Rescorla FJ, et al: Surveillance after initial surgery for pediatric and adolescent girls with stage I ovarian germ cell tumors: report from the Children's Oncology Group. J Clin Oncol 2014; 32(5):465

7. Rescorla FJ, Ross JH, Billmire DF, et al: Surveillance after initial surgery for stage I pediatric and adolescent boys with malignant testicular germ cell tumors: report from the Childrens Oncology Group. J Pediatr Surg 2015; 50(6):1000

8. Shaikh f, Cullen JW, Olson TA, et al: Reduced and compressed cis platin- based chemotherapy in children and adolescents with intermediate risk extra-cranial malignant germ cell tumors: a report from the Childrens Oncology Group J Clin Oncol 2017;35(11):1203

9. Billmire $D$, Vinocur $C$, Rescorla $F$, et al: Outcome and staging evaluation in malignant germ cell tumors of the ovary in children and adolescents: An intergroup study. J Pediatr Surg 2004; 39(3):424

10. Laberge PY, Levesque S: Short-term morbidity and long-term recurrence rate of ovarian dermoid cysts treated by laparoscopy versus laparotomy. J Obstet Gynaecol Can 2006; 28(9):789

11. Madenci AL, Levine $B$, Laufer MR, et al: Poor adherence to staging guidelines for children with malignant ovarian tumors. J Pediatr Surg 2016; 51:1513

12. Vavda Z, Laufer MR, Weldon CB, et al: Diagnostic impact of peritoneal fluid cytology in the setting of pediatric uterine adnexal biopsy or resection. Pediatr Dev Pathol 2016; 19(5):401 
Table 1 Characteristics of 138 girls with primary ovarian tumors COG stage I-III.

\begin{tabular}{|l|c|c|}
\hline Patient Characteristic & & N (\%) or Median (Range) \\
\hline Age at enrollment (Month) & & $153(11-245)$ \\
\hline AFP $(\mathrm{ng} / \mathrm{ml})$ & & $3394.5(2-4233000)$ \\
\hline BHCG (mlU/mL) & & $2(0-999140)$ \\
\hline Tumor Diameter $(\mathrm{cm})$ & & $16(5.5-33.5)$ \\
\hline & & $29(21.0 \%)$ \\
\hline COG Stage & I & $47(34 \%)$ \\
\hline & II & $62(44.9 \%)$ \\
\hline
\end{tabular}

Table 2 Summary of imaging appearance of ovarian tumor for the 133 patients with surgical review.

\begin{tabular}{|l|c|}
\hline Appearance & $\mathbf{N}(\%)$ \\
\hline Cystic & $0(0 \%)$ \\
\hline Cystic with calcification & $0(0 \%)$ \\
\hline Solid & $14(10.5 \%)$ \\
\hline Solid with calcification & $4(3.0 \%)$ \\
\hline Mixed cystic and solid & $78(58.7 \%)$ \\
\hline Mixed cystic and solid with calcification & $33(24.8 \%)$ \\
\hline Unknown & $4(3.0 \%)$ \\
\hline
\end{tabular}

Table 3 Frequency of teratoma elements by imaging appearance of ovarian tumor for the 133 patients with surgical review.

\begin{tabular}{|l|c|c|c|c|c|}
\hline \multirow{2}{*}{ GCT Component(s) } & \multicolumn{5}{|c|}{ Appearance } \\
\cline { 2 - 6 } & Solid & $\begin{array}{c}\text { Solid with } \\
\text { calcification } \\
\mathbf{N = 4}\end{array}$ & Mixed & $\begin{array}{c}\text { Mixed with } \\
\text { calcification } \\
\mathbf{N}=\mathbf{3 3}\end{array}$ & Unknown \\
& 11 & 2 & 42 & 4 & 2 \\
\hline Malignant GCT alone ** & 1 & 2 & 18 & 18 & 1 \\
\hline $\begin{array}{l}\text { Malignant GCT with } \\
\text { mature teratoma } \\
\text { immature teratoma }\end{array}$ & 3 & 0 & 26 & 21 & 2 \\
\hline $\begin{array}{l}\text { Malignant GCT with any } \\
\text { teratoma * }\end{array}$ & $3 / 14$ & $2 / 4$ & $36 / 78$ & $29 / 33$ & $2 / 4$ \\
\hline
\end{tabular}

* Any $\mathrm{T}$ includes those tumors with any element of mature or immature teratoma as some had both types present 